\title{
Successful treatment of childhood onset symptomatic dystonia with levodopa
}

\author{
N A Fletcher, P D Thompson, J W Scadding, C D Marsden
}

\begin{abstract}
Three patients with childhood onset symptomatic dystonia responded to levodopa. None fulfilled criteria for a diagnosis of "dopa responsive dystonia" (Segawa's disease). One may have had athetoid cerebral palsy for almost 25 years. All obtained dramatic and sustained benefit from levodopa therapy. A therapeutic trial of levodopa is advised in all patients in whom dystonia has developed in childhood or early adult life, regardless of suspected aetiology or duration of symptoms.
\end{abstract}

$(\mathcal{F}$ Neurol Neurosurg Psychiatry 1993;56:865-867)

The differential diagnosis of childhood onset dystonia requires the answers to two questions. First, does the patient have idiopathic torsion dystonia (ITD) or a dystonic syndrome secondary to acquired or inherited degenerative neurological disease (symptomatic dystonia)? Second, if the patient has a secondary dystonia can a specific cause be identified and is it treatable? Among the various causes of dystonia ${ }^{1}$ it is essential to consider Wilson's disease and dopa responsive dystonia (DRD) because these conditions are treatable whereas the alternatives are not. Wilson's disease can be excluded by appropriate biochemical tests ${ }^{2}$ but DRD cannot be diagnosed by laboratory investigations. ${ }^{3}$ The diagnosis of DRD depends on a high level of clinical suspicion and a trial of levodopa. Indeed, it has been suggested that such a therapeutic trial is advisable in all cases of dystonia with onset in children or young adults to exclude DRD. ${ }^{4}$ One report stressed the importance of a trial of levodopa in cases of suspected athetoid cerebral palsy which DRD can sometimes resemble. ${ }^{5}$ We report three cases of childhood onset secondary dystonia, who did not have DRD, and in whom levodopa nevertheless induced a dramatic reduction of neurological disability.

\section{Case reports}

Case 1

A 25 year old woman had a lifelong history of rigidity and immobility. She was born six weeks prematurely after an apparently normal pregnancy. There were no neonatal abnormalities and in particular no jaundice. In the first few weeks there were episodes of upward deviation of the eyes and limb stiffening. At the age of one year she could not sit unsupported and showed no signs of starting to walk. Speech and intellect were thought to be normal. A diagnosis of "cerebral palsy" was made. After intensive physiotherapy, she learned to stand when supported by the age of four and later walked between parallel bars. At the age of 14 she gradually lost the ability to stand or walk and inturning of the feet was noted. Subsequently there was relentless deterioration with increasing dysarthria, dysphagia and immobility. For much of the time she was strapped into a wheelchair, unable even to sit unsupported. Striking diurnal variation was noted; in the mornings she could dress, wash and feed herself with difficulty but by midday she was immobile, anarthric and unable to swallow her saliva safely. There was no family history of any neurological disorder and no parental consanguinity.

On examination (at midday), the patient was alert and there were no Kayser-Fleischer (KF) rings. She was unable to sit unsupported. There was a dystonic facial appearance with frequent inappropriate laughter. Optic fundi were normal. There was a supranuclear limitation of ocular movement in all directions, particularly downgaze. There was severe generalised dystonia and minimal voluntary limb movement. Attempted movement was accompanied by occasional myoclonic jerks. Tendon reflexes were all brisk apart from the ankle jerks which were difficult to obtain due to fixed equinovarus contractures of the feet. Plantar responses were flexor and sensation was normal. On general examination she was thin with longstanding bilateral dislocation of the hips.

Routine haematological and biochemical screens including two blood films for acanthocytes, immunoglobulins, protein electrophoresis, copper and caeruloplasmin, creatine kinase, alpha fetoprotein, white cell enzymes, CT brain scan and electroencephalogram were normal. Blood lactate was elevated at $4.73 \mathrm{mmol} / \mathrm{l}$ (normal range $0.5-1.65$ ) and pyruvate $216 \mathrm{umol} / 1$ (normal range 45-160). Phenylalanine and tyrosine levels were normal but the ratio of the phenylalanine concentration (squared) to tyrosine concentration was 133 (normal range 40-66). Psychometry revealed a borderline defective full scale IQ with no evidence of deterioration from a level measured at the referring institution several years previously.

The patient was treated with levodopa (Madopar 62.5 one tablet three times daily) 
with a dramatic improvement within 48 hours. The diurnal fluctuation was abolished so that throughout the day she was able to sit unaided and transfer from bed to wheelchair independently, wash, use the toilet, dress and feed herself without assistance and to speak clearly. This dose of levodopa appeared to be excessive as she initially developed florid chorea which was abolished by reducing the dose of Madopar 62.5 to half a tablet twice daily; this maintained her improvement without involuntary movements. There is mild residual dystonia and she is still unable to walk but the improvement in her condition has been maintained for two years on the same dose of levodopa. The patient has declined any further investigations.

\section{Case 2}

A 23 year old man presented with a history of shaking, muscle stiffness and involuntary movements since childhood. There was no family history of any neurological disorder and no parental consanguinity. He was born prematurely (the exact gestational age was unknown). The labour was "difficult" and forceps were needed. There was marked cyanosis at birth with inability to suck or feed properly for several days; there was no jaundice. He walked at one year but was educationally "slow" at school with no proper speech until the age of five years. At about 14 years, shaking of the hands and an abnormal gait developed. Examination showed a rest tremor of the arms, cogwheel rigidity and a flexed festinating gait. He improved partially with benzhexol (up to $20 \mathrm{mg}$ daily). At the age of 20 his face was noted to be expressionless and there was plastic extrapyramidal rigidity in all four limbs, a rest tremor of the left leg and dystonia of both lower limbs. The optic fundi, cranial nerves, muscle power, tendon reflexes, plantar responses and sensation were normal; there were no KF rings. He walked with great difficulty with a markedly dystonic gait. There was a tendency for his symptoms to become worse during the day but he had not observed any sleep benefit.

An MRI brain scan, full blood count, urea, electrolytes, liver function tests, thyroxine, syphilis serology, white cell enzymes, urinary organic and amino acids, CSF cell count, protein and glucose were all normal or negative. Measurements of biopterins, homovanillic acid and 5 hydroxyindoleacetic acid in the CSF were normal as were serum phenylalanine and tyrosine levels and a tetrahydrobiopterin loading test. ${ }^{6}$ Psychometry showed a verbal IQ of 78 and performance IQ of 76 .

$\mathrm{He}$ was treated with a small dose of levodopa $(50 \mathrm{mg}$ with $12.5 \mathrm{mg}$ of carbidopa daily) with a marked improvement in his symptoms. On this medication he is able to walk almost normally; this benefit has been sustained for over five years although mild residual signs of dystonia in the legs and some akinesia and rigidity have persisted. All attempts to increase the dose of levodopa have led to troublesome dyskinetic movements of the left leg.
Case 3

A 14 year old boy had a history of tremor and involuntary movements since the age of two. His parents were unrelated and there was no family history of neurological disorders. Birth was normal and there was no perinatal jaundice. He walked at 16 months but was persistently unsteady. Single words were spoken at two years although he was always dysarthric. Shaking of the limbs began at the age of two years and became progressively worse. At the age of nine he developed muscle spasms in the back and involuntary movements and postures of the feet. There was a suggestion that his symptoms were less severe in the mornings. He did not learn to read until the age of 14 years.

On examination, there was mild dysarthria and limitation of upgaze but the cranial nerves were otherwise normal; there were no $\mathrm{KF}$ rings and the optic fundi were normal. There were rythmic dystonic movements of the head and limbs with additional dystonia of the feet, arms and trunk which increased when walking. There was no spasticity or extrapyramidal rigidity and muscle power, the tendon reflexes and sensation were normal. The plantar responses could not be assessed because of dystonic posturing of the feet. There were no abnormalities on general examination.

A CT brain scan showed mild cerebellar atrophy but he was unable to tolerate an MRI scan. The full blood count, blood film for acanthocytes, urea, electrolytes, liver function tests, protein electrophoresis, lipids, white cell enzymes, copper, caeruloplasmin and urinary amino and organic acids were normal. The creatine kinase was slightly elevated at 231 IU/1 (normal up to 195).

Treatment with half a Sinemet 125 tablet twice a day was started and he left the UK to return home. The dose of levodopa was gradually increased to one Sinemet 275 tablet three times daily. During the next eight months there was a considerable improvement in his gait with a marked reduction in the involuntary movements. He was able to perform all activities of daily living easily and was able to play football and to socialise with his peers. No further investigation or follow up has been possible because the patient lives abroad.

\section{Discussion}

The cases described in this report all failed to meet the diagnostic criteria for ITD which specify a purely dystonic neurological disorder, normal birth history and early developmental milestones and no evidence of a predisposing cause for the dystonia either on clinical or laboratory grounds. ${ }^{7}$ Features such as oculogyric crises, seizures and grossly delayed motor development, diurnal variation of symptoms, abnormal eye movements, Parkinsonism, and intellectual impairment which were seen in these patients in varying combinations are not consistent with a diagnosis of ITD. Abnormal central dopaminergic 
activity has been detected in some patients with $\mathrm{ITD}^{8}$ but significant responsiveness to levodopa is not a feature of ITD. Although all three clearly had secondary dystonia, in none could a more specific diagnosis be established with confidence. Case 1 had been diagnosed as having athetoid cerebral palsy but this was unlikely. The clinical features strongly resembled those seen in patients with biopterin synthesis defects ${ }^{9-11}$ and this was not entirely excluded by the normal phenylalanine level. ${ }^{9}$ The lactic acidosis raised the possibility of Leigh's disease or a mitochodrial encephalomyopathy ${ }^{12}$ but the patient declined all further investigations and the diagnosis could not be refined further. Cases 2 and 3 had no specific diagnostic clues and a definitive diagnosis was not possible in either. However, their most important common clinical feature was remarkable improvement after a short period of levodopa therapy; two required only very small doses, and in all three this improvement has been sustained.

DRD (Segawa's disease) is characterised by an onset in childhood or adolescence of dystonia, mainly affecting the legs, with or without other features such as diurnal fluctuation, Parkinsonism and brisk tendon reflexes. The salient diagnostic feature of DRD is extreme sensitivity to small doses of levodopa which produces complete and sustained resolution of neurological deficits even after many years of disability. ${ }^{313}$ The reason for this remarkable sensitivity to levodopa is unclear but abnormalities of central dopaminergic function have been detected by positron emission tomography in DRD. ${ }^{14}$ Because of such a dramatic response to treatment, rather variable clinical features and the lack of a specific diagnostic test, a trial of levodopa is advised in all children and young adults with dystonia ${ }^{4}$ including athetoid (that is, dystonic) cerebral palsy ${ }^{5}$ specifically to exclude DRD. A diagnosis of DRD is highly unlikely in the cases we describe since they exhibited clinical features (such as mental retardation and prominent myoclonus) not typical of DRD. However, the most important feature which separates these cases from the syndrome of DRD is the nature of the response to levodopa. Although this was functionally very useful in each case, it was incomplete and left residual deficits. In addition, the prominence of and sensitivity to levodopa induced dyskinesias are quite different from the response seen in DRD. Nevertheless, all obtained functionally very useful clinical improvement with levodopa therapy. These cases show clearly that significant levodopa responsiveness in cases of dystonia is not confined to DRD. Consequently, they illustrate the important point that a trial of levodopa is essential in all patients developing dystonia in childhood or early adult life, even if the clinical features are atypical for DRD and consistent with another condition. Clearly, this recommendation applies to patients with suspected athetoid cerebral palsy; such patients account for up to $25 \%$ of all cases of cerebral palsy and one of our patients had been thought to have this condition for almost 25 years. It is possible that there are similar patients who may respond to levodopa therapy and a therapeutic trial is strongly advised in all such cases, regardless of duration of symptoms.

Although our cases illustrate that there are levodopa responsive dystonic disorders other than classical DRD (Segawa's disease), the nature of these is not clear. We suspected that case 1 may have had a biopterin synthesis defect but this could not be established. Certainly, the diurnal fluctuation of symptoms was strongly suggestive as was the history of oculogyric crises and mental retardation. ${ }^{9}$ Although such a metabolic defect was not found in case 2 , abnormalities of biopterin metabolism should be considered in similar patients. Other patients with biopterin synthesis defects have shown a marked but incomplete response to levodopa ${ }^{1011}$ similar to that seen in our cases.

1 Marsden $\mathrm{CD}$. Investigation of dystonia. In: Fahn $\mathrm{S}$, Marsden CD, Calne DB, eds. Dystonia 2. Advances in neurology, vol 50. New York: Raven Press, 1988:35-44.

2 Sternlieb I, Giblin DR, Scheinberg IH. Wilson's Disease. In: Marsden CD, Fahn S, eds. Movement disorders 2. London: Butterworths, 1987;288-302.

3 Nygaard TG, Marsden CD, Duvoisin RC. Dopa responsive dystonia. In: Fahn S, Marsden CD, Calne DB, eds. Dystonia 2. Advances in neurology, vol 50. New York: Raven Press, 1988:377-85.

4 Marsden CD, Quinn NP. The dystonias. BMF 1990;300: 139-44.

5 Boyd K, Patterson V. Dopa responsive dystonia: a treatable condition misdiagnosed as cerebral palsy. $B M \mathcal{F}$ 1989;298:1019-20.

6 Smith I, Leeming RJ, Cavanagh NPC, Hyland K. Neurological aspects of biopterin metabolism. Arch Dis Childhood 1986;61:130-7.

7 Marsden CD, Harrison MJG. Idiopathic torsion dystonia (Dystonia musculorum deformans): a review of fortytwo patients. Brain 1974;97:793-810.

8 Playford ED, Sawle GV, Fletcher NA, Marsden CD, Brooks DI. The nigrostriatal dopaminergic system in familial idiopathic torsion dystonia. Neurology 1991; 41(suppl 1):273.

9 Smith I, Howells DW, Hyland K. Pteridines and monoamines: Relevance to neurological damage. Postgrad Med ₹ 1986;62:113-23.

10 Tanaka K, Yoneda M, Nakajima $T$, et al. Dihydrobiopterin synthesis defect: An adult with diurDihydrobiopterin synthesis defect: An adult with diur-

11 Fink JK, Barton N, Cohen W, et al. Dystonia with marked diurnal variation associated with biopterin deficiency.
, et al Neurology 1988;38:707-11.

12 Evans OB, Bock HO, Parker CC, Hanson RR. Inborn errors of metabolism of the nervous system. In: Bradley WG, Daroff RB, Fenichel GM, Marsden CD, eds. Neurology in clinical practice. Boston: ButterworthHeinemann, 1991:1269-1322.

13 Nygaard TG, Duvoisin RC. Hereditary dystonia parkinsonism syndrome of juvenile onset. Neurology 1986;36:1424-28.

14 Sawle GV, Leenders KL, Brooks DJ, et al. Dopa responsive dystonia: $18 \mathrm{~F}$ dopa positron emission tomography. Ann Neurol 1991;30:24-30. 